\title{
Pulmonary Metastases in Pseudomyxoma Peritonei Syndrome
}

\author{
Keith D. Mortman, MD, Paul A. Sugarbaker, MD, Barry M. Shmookler, MD, \\ Vicente C. DeGuzman, MD, and Mark S. Soberman, MD
}

Section of Thoracic Surgery, Department of Surgery; Department of Pathology; and Sections of Thoracic Oncology and Surgical Oncology, Washington Cancer Institute, Washington Hospital Center, Washington, DC

Background. Pseudomyxoma peritonei (PMP) is a rare disease arising from a mucinous cystadenoma of appendiceal origin. The syndrome has been characterized by progressive growth of mucinous tumors, tense mucinous ascites, and ultimately death. Abdominal and pelvic recurrence after resection of intraperitoneal disease occurs in all patients unless adjunctive measures are taken. Local spread of PMP by direct extension to the pleural or pericardial space is uncommon but has been reported in the literature. Here we report development of pulmonary parenchymal metastases after treatment for PMP.

Methods. The charts of 3 patients were retrospectively

$\mathrm{P}$ seudomyxoma peritonei (PMP) has been a recognized clinical entity since its description by Werth in 1884 [1]. However, because it is a rare disease, a precise description of its clinical and pathologic features has not been available until recently [2,3]. A full description of the appendiceal adenoma, progression of disease as a result of appendiceal rupture, and a presentation of the natural history of disease progression now constitute the PMP syndrome [4]. In recent reports, Ronnett and colleagues $[5,6]$ described the histopathology of the PMP syndrome and proposed reproducible clinical and pathologic guidelines for the diagnosis that permitted delineation of statistically significant prognostic groups. Despite extensive and massive progression within the abdomen and pelvis, extraabdominal metastasis of PMP has been a rare occurrence. There are sporadic case reports of direct extension into the pleural and pericardial spaces [7]. Herein, we present 3 patients with pulmonary parenchymal metastases of PMP.

\section{Patient 1}

A 47-year-old white female nonsmoker with PMP of appendiceal origin underwent a cytoreductive operation and heated intraperitoneal chemotherapy in January 1995. Her immediate postoperative course was unremarkable. A routine follow-up computed tomographic scan of the chest in April 1995 revealed pulmonary nodules of $1.0 \mathrm{~cm}$ and $1.2 \mathrm{~cm}$ in the right lower lobe.

Accepted for publication May 2, 1997.

Address reprint requests to Dr Soberman, Section of Thoracic Surgery, Washington Hospital Center, 110 Irving St, NW, Washington, DC 20010. reviewed for the presentation and management of metastatic PMP.

Results. Three patients underwent resection for pulmonary parenchymal metastases of PMP. All patients recovered uneventfully. They continue to do well after 2 to 8 years of follow-up.

Conclusions. Pulmonary metastasectomy for PMP is safe and effective after treatment of intraperitoneal disease.

(Ann Thorac Surg 1997;64:1434-6)

(C) 1997 by The Society of Thoracic Surgeons

There was no evidence of mediastinal adenopathy or pleural involvement. The presumptive diagnosis was metastatic mucinous tumor of the appendix.

The patient underwent a right lower lobectomy and lymph node dissection. The pulmonary nodules were described pathologically as metastatic low-grade mucinous tumor consistent with the previously diagnosed PMP. There was no evidence of lymphatic metastases.

The patient did well and was discharged home on the fifth postoperative day. She continues to do well with no further evidence of disease.

\section{Patient 2}

A 48-year-old white man underwent appendectomy in August 1989 for a perforated mucinous appendiceal tumor. The patient subsequently experienced PMP and had cytoreductive operations in May 1990 and February 1992. In early 1994 a computed tomographic scan revealed a pulmonary nodule. A repeat computed tomographic scan in March 1995 revealed progression of the lesion. Because of progressive intraperitoneal disease, the patient was admitted again in July 1995 for a cytoreduction procedure and intraperitoneal chemotherapy. Chest computed tomography at this time revealed a $4.0 \times$ $3.5 \times 3.0-\mathrm{cm}$ mass in the superior segment of the left lower lobe (Fig 1). There was no mediastinal adenopathy or evidence of pleural disease. After recovery from his cytoreductive operation and during the same hospitalization, the patient underwent left lower lobectomy and mediastinal lymph node dissection. Histologic examination of the parenchymal mass showed a focus of metastatic well-differentiated mucinous adenocarcinoma con- 


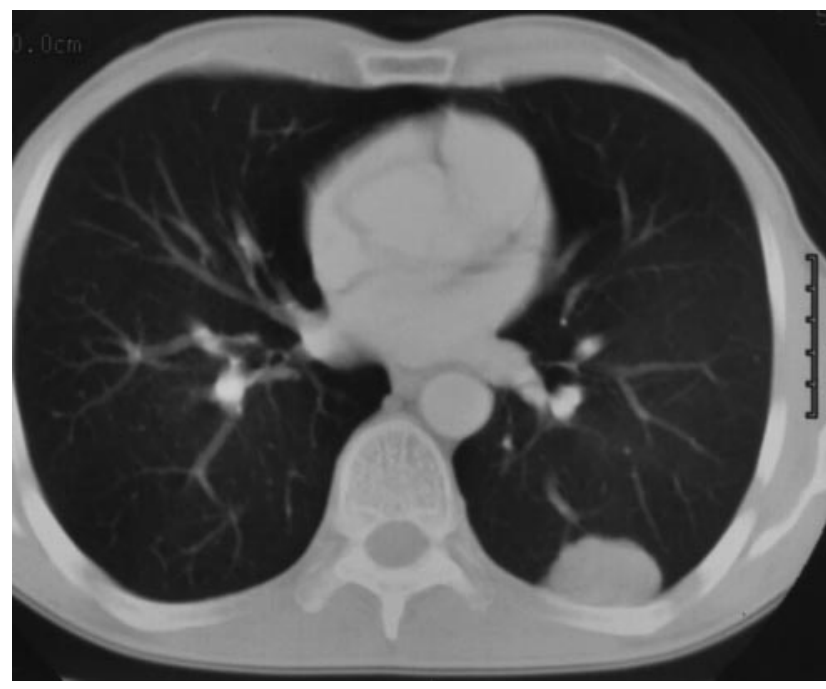

Fig 1. Chest computed tomographic scan demonstrating left lower lobe metastasis from pseudomyxoma peritonei.

sistent with the patient's previous gastrointestinal primary (Fig 2).

The patient was discharged after an unremarkable postoperative course. He remains without clinical evidence of disease at this time.

\section{Patient 3}

A 41-year-old white male nonsmoker with PMP of appendiceal origin had a cytoreductive operation in June 1987 followed by systemic chemotherapy. During his postoperative course, an abnormality was noted in the right lung base on chest radiograph. Computed tomography of the chest revealed a $3.5-\mathrm{cm}$ soft tissue mass in the right lower lobe as well as a $1-\mathrm{cm}$ nodule in the right upper lobe. There was no pleural effusion or mediastinal adenopathy. Computed tomography also showed a small

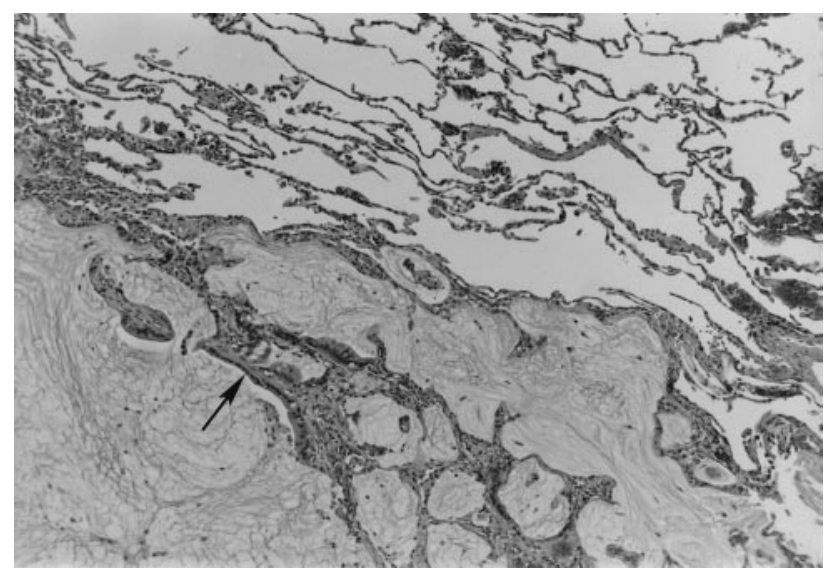

Fig 2. Section of lung demonstrating metastatic mucinous tumor of appendiceal origin. Note the abundant mucinous material containing sparse strips of bland simple columnar epithelium (arrow). (Hematoxylin and eosin; $\times 20$ before $50 \%$ reduction.) nodule anterior to the right lobe of the liver that was contiguous with the diaphragm. In October 1989, the patient had wedge resections of a $1 \times 1 \times 2-\mathrm{cm}$ right upper lobe nodule and a $4 \times 5 \times 1-\mathrm{cm}$ right lower lobe nodule. The diaphragmatic mass was also resected.

The patient was discharged on postoperative day five after an uneventful recovery. Follow-up computed tomography performed in April 1990 showed no evidence of pulmonary nodules, mediastinal adenopathy, or pleural effusion. The patient remains alive and well.

\section{Gross and Microscopic Pathology}

The resected specimens contained one to a few intraparenchymal well-circumscribed gelatinous or mucoid nodules ranging from 1.5 to $3.5 \mathrm{~cm}$ in maximum dimension. Microscopically, the lesions revealed a smooth, pushing interface with the adjacent pulmonary alveoli. There was a predominance of mucinous substance containing scattered strips of simple columnar epithelium with only focal mild atypia (Fig 2). The histologic features of these metastatic nodules were essentially identical to the primary appendiceal tumor. Hilar and mediastinal lymph nodes revealed anthracosis and reactive lymphoid hyperplasia but contained no mucinous neoplasm.

\section{Comment}

Local recurrence of intraabdominal mucinous tumors and ascites is always seen after surgical treatment alone for PMP syndrome [8]. Distant hematogenous metastasis of PMP, however, has not been confirmed. Those cases with pleural or pericardial involvement are believed to be caused by the migration of tumor cells through the diaphragm. Because of the large number of PMP patients treated at this institution and long-term survival occurring as a result of locally aggressive treatments, new information about this rare disease is becoming available. A review of the world's literature yielded 1 case of pulmonary parenchymal metastasis of PMP described as a postmortem discovery in 1962 [9]. We hypothesize that these cases of pulmonary PMP are the result of hematogenous spread rather than suspected migration across the diaphragm, since no evidence of pleural disease was present in 2 of the patients. It is unclear whether these metastases represent true angioinvasion or are secondary to extensive intraoperative manipulation of tumor and mucinous tumor emboli. As only 3 of the more than 100 PMP patients we have treated have had pulmonary metastases, no distinctive histologic features have, thus far, been predictive of metastatic potential. Aggressive cytoreductive surgical procedures and intraperitoneal chemotherapy may modify the natural history of PMP, making the occurrence of distant metastases more likely. Pulmonary metastasectomy has been accepted as effective therapy for various tumors metastatic to the lung. Further follow-up will be necessary to delineate the long-term effectiveness of pulmonary resection for metastatic PMP. 


\section{References}

1. Werth R. Pseudomyxoma peritonei. Arch Gynakol 1884;24: 100-18.

2. Fann JI, Vierra M, Fisher D, Oberhelman HA, Cobb L. Pseudomyxoma peritonei. Surg Gynecol Obstet 1993;177: 441-7.

3. Sugarbaker PH, Kern K, Lack E. Malignant pseudomyxoma peritonei of colonic origin: natural history and presentation of a curative approach to treatment. Dis Colon Rectum 1987;30: 772-9.

4. Sugarbaker PH, Ronnett BM, Archer A, et al. Pseudomyxoma peritonei syndrome. Adv Surg 1997;30:233-80.

5. Ronnett BM, Zahn CM, Kurman RJ, Kass ME, Sugarbaker PH, Shmookler BM. Disseminated peritoneal adenomucinosis and peritoneal mucinous carcinomatosis: a clinicopathologic analysis of 109 cases with emphasis on distinguishing patho- logic features, site of origin, prognosis, and relationship to "pseudomyxoma peritonei." Am J Surg Pathol 1995;19:1390408.

6. Ronnett BM, Kurman RJ, Zahn CM, et al. Pseudomyxoma peritonei in women: a clinicopathologic analysis of 30 cases with emphasis on site of origin, prognosis, and relationship to ovarian mucinous tumors of low malignant potential. Hum Pathol 1995;26:509-24.

7. Mets T, Van Hove W, Louis H. Pseudomyxoma peritonei. Chest 1977;72:792-4.

8. Wertheim I, Fleischhacker D, McLachlin CM, Rice LW, Berkowitz RS, Goff BA. Pseudomyxoma peritonei: a review of 23 cases. Obstet Gynecol 1994;84:17-21.

9. Berge T. "Mucocele appendicis" with pseudomyxoma peritonei and pulmonary metastases. Acta Pathol Microbiol Scand 1964;60:483.

\section{Important Announcement}

In addition to postal services and FAX, The Annals of Thoracic Surgery Editorial Office may now be reached by e-mail. The address is:

\section{ats@wudos2.wustl.edu}

The Annals of Thoracic Surgery Web site addresses are:

\section{http://www.sts.org/annals/ http://www.ctsnet.org/journals/}

